\title{
PERFORATED APPENDIX IN THE INDIRECT INGUINAL HERNIAL SAC PRESENTING WITH A SCROTAL EMERGENCY: A PROSPECTIVE STUDY
}

\author{
Purujit Choudhury 1 \\ ${ }^{1}$ Associate Professor, Department of Surgery, Gauhati Medical College.
}

\begin{abstract}
BACKGROUND

Amyand's hernia is a rare form of inguinal hernia, in which an inflamed appendix is incarcerated in a hernial sac. The clinical presentation of Amyand's hernia varies depending on the extent of inflammation involved in the hernial sac and the presence or absence of a scrotal abscess. If a scrotal abscess is present, this usually indicates that the appendix in the hernial sac is perforated. However, without the availability of Computed Tomography (CT) scans, the condition is often preoperatively misdiagnosed as a strangulated inguinal hernia, testicular torsion or vascular obstruction. Emergency treatment is mandatory if perforated appendix in inguinal hernia sac.
\end{abstract}

\section{METHOD}

25 male patients aged from 20-35 yrs. were studied who were presented with diffuse abdominal pain more in the right lower abdomen, nausea and vomiting followed by feature of acute scrotum. All the patients were admitted in the emergency surgical ward. Laboratory and radiological evaluation suggested presence of inactive, pathology in the inguino-scrotal region. All the patients were resuscitated and all necessary investigations were advised including ultrasonography; 5 patients were advised for CT scan of the whole abdomen including inguinoscrotal region. All other diseases resemble the features of appendicular perforation in the hemiscrotum including torsion of testes were excluded.

\section{RESULT}

Patients were resuscitated immediately after admission. Surgical exploration on the next day revealed patent right processus vaginalis with purulent collection resulting from the presence of perforated tip of appendix in the hernial sac. Appendicectomy with drainage of scrotal collection and ligation of hernial sac with modified Bassini's herniorrhaphy without mesh led to satisfactory recovery of all the patients in this study. McBurney's exploration was done and toileting of peritoneal cavity with application of drain, those presenting features of peritonism.

\section{CONCLUSION}

Generally, the incidence of having a normal appendix within an inguinal hernial sac is about $1 \%$, whereas only $0.1 \%$ of all cases of appendicitis present in an inguinal hernia, further underscoring the rarity of the condition. Though Amyand's hernia is rare, it is not unusual. Perforated appendix in hernia sac, eventually presenting with hemiscrotum mimic torsion of testes. Proper investigation like CT/US can detect easily. Resuscitation followed by emergency surgical exploration is the treatment of choice.

\section{KEYWORDS}

Acute Scrotum, Appendicular Perforation, Amyand's Hernia.

HOW TO CITE THIS ARTICLE: Choudhury P. Perforated appendix in the indirect inguinal hernial sac presenting with a scrotal emergency: a prospective study. J. Evolution Med. Dent. Sci. 2016;5(47):2988-2992, DOI: 10.14260/jemds/2016/697

\section{INTRODUCTION}

Acute appendicitis sometimes manifests with rare clinical features owing to its unusual anatomic locations. The scrotum develops as a part of the abdominal cavity and the processus vaginalis remains patent $80-90 \%$ of newborns and gradually declines to $15-37 \%$ during adulthood. Estes descend towards scrotum, which is directed by gubernaculums and processus vaginalis. It is nothing but fold of peritoneum. It reaches the bottom of scrotum at 9 months of gestation. It descends through deep inguinal ring, then traverses inguinal canal, then superficial inguinal ring and ultimately rest on bottom of scrotum.

Financial or Other, Competing Interest: None.

Submission 13-03-2016, Peer Review 09-04-2016,

Acceptance 14-04-2016, Published 11-06-2016.

Corresponding Author:

Dr. Purujit Choudhury,

P.O. Gopinath Nagar,

Arya Path,

Gauhati-781016,

Assam.

E-mail: drpurujit@yahoo.in

DOI: 10.14260/jemds/2016/697
Normally, most of the newborn have two obliterations at two levels, one at the level of deep ring and one at the superior pole of testes. In between two, the obliterated processus remain as content of spermatic cord. Below superior pole of testes, the processus become known as tunica vaginalis which has two layers. When processus remain patent, then indirect hernia has to occur and right-sided inguinal hernia is commoner due to deferred descend of right testis. Though appendix is very rare to be the content of the hernia sac, yet it is not impossible. When it became the content, then every chance to be strangulated result in gangrene and perforation might lead to different catastrophe including hemiscrotum syndrome.

Because internal ring is very narrow under tight sphincter control of two complete crura of internal oblique. Moreover, appendicular artery and posterior caecal artery, the artery supply of appendix are end arteries. Both are from lower division of ileo-colic branch of superior mesenteric artery. Scrotal symptoms as manifestations of acute appendicitis are infrequent; 25 male patients were studied who were presenting with features of acute appendicitis with scrotal oedema, pain and signs of inflammation. Right more than left. 
Scrotal more troublesome than abdomen. From surgical point of view pelvic position is about $20-25 \%$, which is next to retrocaecal (74\%). Our tertiary centre is a tertiary centre and Medical College. Hence, input of different types of acute appendicitis and inguinal hernia of different varieties and presentation in emergency setup is not less than optimum for research and study. Amyand's hernia is said to be rare type of hernia, where content of the hernial sac is vermiform appendix. There is every possibility that this type of content to get strangulated. Hence, perforation and abscess. Patients may present with inguinoscrotal swelling, which is much tender mimic testicular torsion. Scrotum is swollen, tender and acutely inflamed. Amyand's Hernia ( $\mathrm{AH})$ is a rare variety of inguinal hernias containing vermiform appendix.[1] It is named after a Sergeant Surgeon to King George II of England, Dr. Claudius Amyand, who first described the presence of a perforated appendix in an inguinal hernia sac in 11 years old boy in the 18th century; $1 \%$ of all hernias in the region is Amyand.

These are either bubonocoele, funicular or complete variety of inguinal hernia. Inflamed right hemiscrotum with intense pain and features of shock like syndrome with much toxicity are prominent in complete variety. The underlying mechanisms that can lead to acute appendicitis within an Amyand's hernia include the reduction of the appendix's blood supply due to adhesions that may cause non-reducibility of the hernia and compression in the external ring originating from increase in intra-abdominal pressure. These factors lead to recurrent inflammation and bacterial overgrowth. Jeopardised vascular supply might lead to catastrophic features needs urgent surgical intervention. Delayed treatment may terminate to fatal outcome.

\section{MATERIALS AND METHODS}

25 male patients admitted at Gauhati Medical College in emergency ward from November 2012 till March 2015 were studied. Their age group between 20 yrs. to 35 yrs. presented with a one-day history of right scrotal pain.[1] and swelling, diffuse right lower abdominal pain, nausea, vomiting and lowgrade fever. Five patients had diffuse involvement of scrotum with lower right abdominal swelling and tender abdomen with rigidity and positive rebound tenderness. They were advised immediate CT scan, Figure 3, evaluation of whole abdomen. There was no history of injury, but history of occasional inguinoscrotal swelling specially during coughing with spontaneous disappearance. On examination the patient appeared toxic, febrile and anaemic with dehydration, off and on nausea and vomiting; often complaining of nausea, frequency of micturition and bowel increases. Local examination revealed tender, oedematous, erythematous right hemiscrotum (Left scrotum slightly involved in mild-tomoderate intensity) Figure 2 with raised local temperature.

17 patients showed tender inguinoscrotal swelling with soft consistency in the inguinal region. It is due to abscess formation following perforation. Pain in the scrotum is intractable and increased during movement. Local rise of temperature present. The cremasteric reflex was absent. Abdominal examination showed a soft, non-distended abdomen with mild tenderness in the right iliac fossa near the deep inguinal point.[2] The patients were complaining of more pain and more discomfort in the scrotum than abdomen.
Investigation showed - Haemoglobin 9.2-11 gm\%, WBC16300-21000/cmm (Polymorph 96\%) normal renal and liver function tests. Plain picture abdomen showed no abnormalities. Colour Doppler study of right scrotum showed increased vascularity of the right testis. Ultrasonography of the abdomen showed presence of fluid with debris and internal echoes in the right hemiscrotum and inguinal canal.[2] No evidence of torsion of the testes. CT whole abdomen showed paralytic ileus due to peritonism in 5 patients. Infected pus may be the aetiology. Those patients presented much more febrile, toxic and restless. Features of pre-septicaemia with high total count.

Surgical exploration of the inguino-scrotal region revealed a patent processus vaginalis containing purulent fluid with feculent smell. There was evidence of epididymo-orchitis and the spermatic cord was oedematous and thickened. The tip of the appendix was in the hernial sac, projecting about $3 \mathrm{~cm}$ through the internal inguinal ring. The tip of the appendix was perforated in the sac. Laparotomy through right lower paramedian incision revealed a long inflamed appendix Fig. 1 of all the cases. ${ }^{[3]}$ extending into the patent processus vaginalis through the deep inguinal ring. Appendicectomy was performed in all the cases.[3,4] The right hemiscrotum and peritoneal cavity were irrigated and herniorrhaphy was performed by modified Bassini. No mesh applied. The postoperative recovery was uneventful. The patients were doing well in postoperative period. Follow-up for last 9 months reveals no abnormalities.

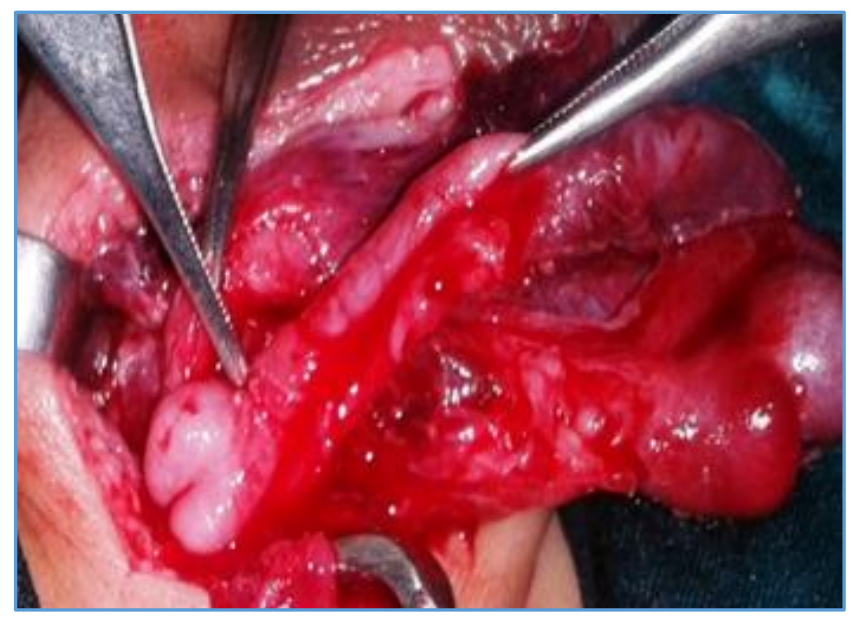

Fig. 1: Inflamed Appendix with Perforation in the Scrotal Sac

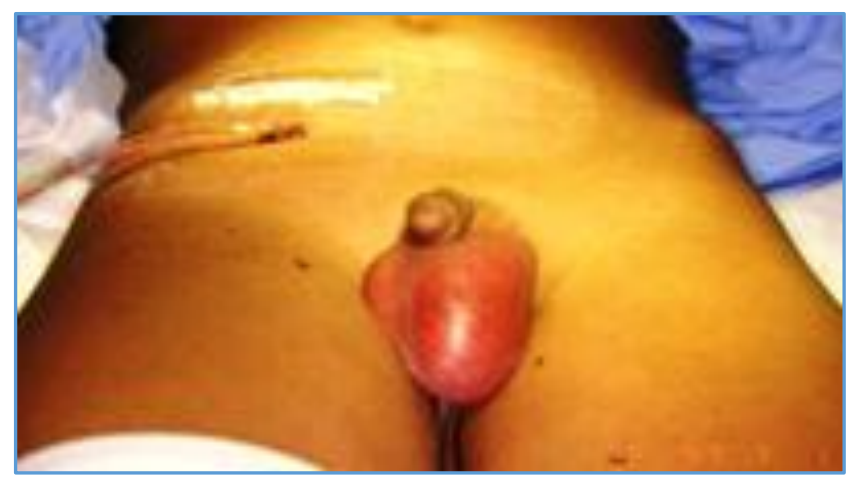

Fig. 2: Drain after Appendicectomy-Scrotum Inflamed 

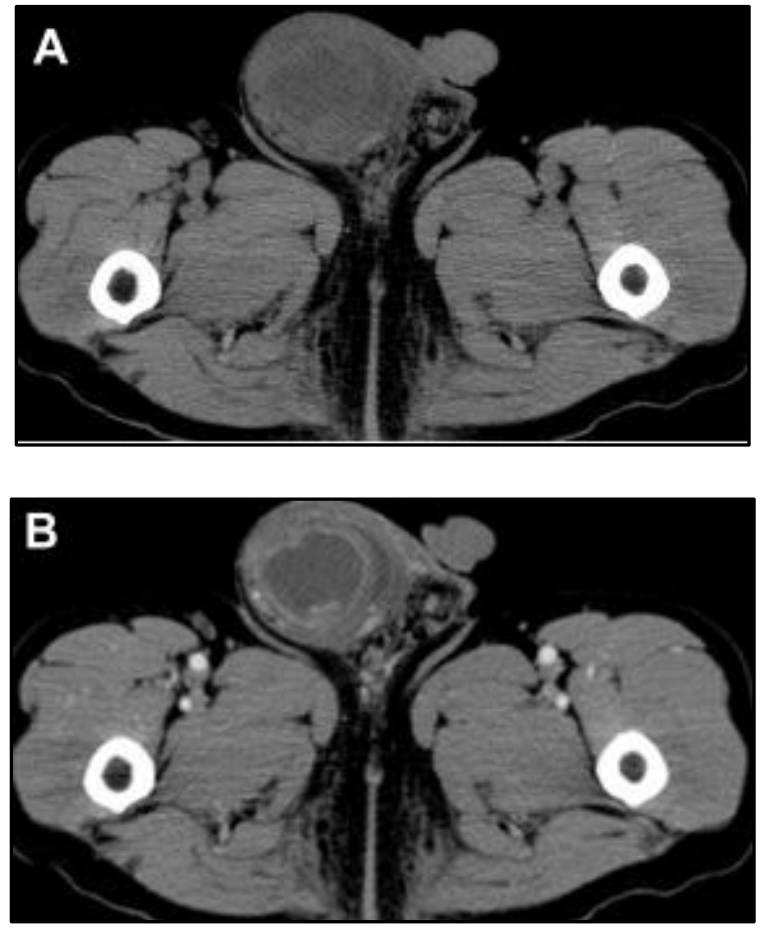

Fig. 3: CT (A) Non-Contrast (B) Contrast Enhance. Right Scrotum Enlarged, Oedema, Wall Thickened, Localized Fluid Collection with Rim Enhancement Consistent with Abscess

\section{DISCUSSION}

Acute scrotum.[4] in younger age can be caused by a number of clinical conditions including testicular torsion, epididymoorchitis, torsion of testicular appendages, infected hydrocele, incarcerated inguinal hernia and less commonly by thrombosed scrotal veins, Henoch-Schonlein purpura and fluid leakage from VP shunt. Acute scrotum secondary to acute appendicitis.[5] is rare in young and children. All such cases have patent processus vaginalis as in my case.

Acute appendicitis within an inguinal hernia accounts for $0.1 \%$ of all cases. Inflammation of the appendix is attributed to external compression of the appendix at the neck of the hernia. The inflammatory status of the vermiform appendix determines the surgical approach and the type of hernia repair. All surgeons agree that if appendicitis exists, the repair of the hernia should be performed with Bassini or Shouldice techniques without making use of synthetic meshes or plugs within the defect due to the high risk of suppuration of such materials. In the case of a normal appendix, incidentally found within the hernial sac, the performance of a prophylactic appendectomy along with the hernia repair is not favoured by many authors. Appendectomy adds the risk of infection to an otherwise clean procedure.

Superficial wound infection increases morbidity and deep infection may contribute to hernia recurrence. In addition, surgical manipulation to achieve visualization of the entire appendix and its base by enlarging the hernial defect or distending the neck of the hernial sac increases the possibility of recurrence by weakening the anatomic structures around the defect. There are authors who recommend reduction of the appendix and mesh hernioplasty if there is no acute appendicitis and appendectomy followed by endogenous hernia repair if an inflamed appendix is found. Although these general rules are certainly acceptable, there are more clinical scenarios to keep in mind. Losanoff and Basson have distinguished four basic types of Amyand's hernias, which should be treated differently as -

Classification of Amyand's Hernias after Losanoff and Basson

\begin{tabular}{|c|c|c|}
\hline Classification & Description & $\begin{array}{c}\text { Surgical } \\
\text { Management }\end{array}$ \\
\hline Type 1 & $\begin{array}{c}\text { Normal appendix } \\
\text { within inguinal } \\
\text { hernia sac }\end{array}$ & $\begin{array}{l}\text { Hernia reduction } \\
\text { with mesh, } \\
\text { appendicectomy in } \\
\text { young }\end{array}$ \\
\hline Type 2 & $\begin{array}{c}\text { Acute } \\
\text { appendicitis } \\
\text { within inguinal } \\
\text { hernia with no } \\
\text { abdominal sepsis }\end{array}$ & $\begin{array}{l}\text { Appendicectomy } \\
\text { through hernia, } \\
\text { primary endogenous } \\
\text { repair of hernia, no } \\
\text { mesh }\end{array}$ \\
\hline Type 3 & $\begin{array}{c}\text { Acute } \\
\text { appendicitis in } \\
\text { inguinal hernia } \\
\text { with abdominal } \\
\text { and peritoneal } \\
\text { sepsis } \\
\end{array}$ & $\begin{array}{l}\text { Laparotomy, } \\
\text { appendicectomy, } \\
\text { primary repair of } \\
\text { hernia, no mesh }\end{array}$ \\
\hline Type 4 & $\begin{array}{c}\text { Acute } \\
\text { appendicitis } \\
\text { within inguinal } \\
\text { hernia, related or } \\
\text { unrelated } \\
\text { abdominal } \\
\text { pathology }\end{array}$ & $\begin{array}{c}\text { Manage as type } 1 \text { or } \\
2 \text { hernias. Deal } 2^{\text {nd }} \\
\text { pathology }\end{array}$ \\
\hline
\end{tabular}

The absence of inflammation in Type 1 advocates elective hernioplasty. Using a prosthetic material in such cases carries the expectation of improved longevity of the repair. It avoids tension on the suture lines and circumvents the metabolic problems related to collagen deficiency, which is known to exist in hernia patients. Whether to remove or leave behind a normal appendix in this clinical scenario cannot be determined, because no evidence-based information exists. The decision is rather based on common sense relating to the patient's age, life expectancy and life-long risk of developing acute appendicitis and the size and overall anatomy of the appendix. Paediatric or adolescent patients have a significantly higher risk of developing acute appendicitis and should therefore have their appendices removed, compared to middle-aged or elderly individuals in whom the appendix should probably be left intact.

Long, curved appendices have a higher risk of inflammation. [5] Additionally a long appendix, which stretches the caecum may cause chronic pain if left behind. Manipulations to detach and reduce the appendix in the abdomen may stimulate the inflammatory process. Furthermore, consideration of appendectomy.[6] in young patients must take into account the size of the hernia, since prosthetic material is contraindicated but large hernias are more likely to recur if repaired by making use of endogenous tissue only.

The decision is easier in Type 2 hernias, where appendicitis is found, as they should be treated with appendectomy; however, the hernia repair should be performed without making use of prosthetic materials. On the other hand, in septic patients with Amyand's hernia.[7,8] Type 
3 (Acute appendicitis with peritonitis) or Type 4 (Acute appendicitis with other pathology), even the hernioplasty may be contraindicated if the patient's condition is poor or life expectancy is limited.

Yu-Hsiu Juan reported that Amyand's hernia.[9] is a rare form of inguinal hernia, in which an inflamed appendix is incarcerated in a hernial sac. The clinical presentation of Amyand's hernia varies depending on the extent of inflammation involved in the hernial sac.[10] and the presence or absence of a scrotal abscess. If a scrotal abscess is present, this usually indicates that the appendix in the hernial sac is perforated. However, without the availability of Computed Tomography (CT) scans, the condition is often preoperatively misdiagnosed as a strangulated inguinal hernia.

Claudius Amyand was a British Surgeon who, in 1735, performed the first successful appendectomy from an inguinal hernia in which the inflamed appendix was contained in a right inguinal hernia in an 11-year-old boy. The term Amyand's hernia was defined as an uncommon inguinal hernia that contains the appendix which may be perforated, inflamed or normal. The presentation of an Amyand's hernia can vary depending on its inflammatory extent and closely resembles that of a strangulated inguinal hernia. The diagnosis is difficult to make preoperatively. If there is a scrotal abscess within the hernial sac, a perforated appendix is usually indicated. In a review of the literature, only Salemis et al reported a case of Amyand's hernia.[10] complicated by a peri-appendicular abscess. However, a preoperative Computed Tomography (CT) scan was not performed and the patient was misdiagnosed with a strangulated hernia.

A non-inflamed appendix within an inguinal hernia is uncommon with the reported incidence varying from $0.28 \%$ to $1 \%$. The incidence of inguinal hernia containing an inflamed appendix is rare, estimated at $0.07-0.13 \%$. Amyand's hernia usually occurs in the older population ( $>60$ years), but a smaller number have presented in children. Most cases occur on the right side, reflecting the usual intra-abdominal position of the organ and the fact that right-sided inguinal hernias are more common than left-sided hernias. Although a handful of left-sided Amyand's hernias have been reported.[11], these may be associated with situs inversus, intestinal malrotation, or a mobile caecum. The most common presentation of Amyand's hernia is a right inguinal mass that is inflamed and tender with variable degrees of abdominal pain and vomiting. The classic signs of appendicitis including a fever and leucocytosis are often absent.

Preoperative diagnosis requires a high degree of clinical suspicion and awareness and is extremely difficult, because the symptoms are non-typical and depend on the extent of peri-appendicular inflammation and the presence or absence of peritoneal contamination and usually point to incarcerated or strangulated hernia. In 2009, Coleman et al reported a case of incarcerated appendicitis masquerading as epididymitis. The final diagnosis was revised as an Amyand's hernia after performance of an abdominal CT scan. Fernando and Leelaratna and Losanoff and Basson defined Amyand's hernia as an inguinal hernia containing (1) A non-inflamed appendix, (2) An inflamed appendix, (3) A perforated appendix, or (4) Acute appendicitis complicated with related or unrelated intra-abdominal pathology. The classification systems have been based on the surgical treatment recommended, which differs for each type of Amyand's hernia.
CT has been used in the preoperative evaluation of incarcerated inguinal hernias and was diagnostic of Amyand's hernia in many cases. Luchs et al were the first to describe cases (Two) on CT scans. Constantine illustrated four subtypes of Amyand's hernia by abdominal CT images. He suggested that abdominal CT is particularly invaluable in the recognition of types 3 and 4, which are more complicated and diverse than types 1 and 2 . The sagittal and coronal reformats are particularly useful in identifying the blind-ending tubular appendix arising from the caecum and entering the inguinal canal. This was exemplified in 2007 by Gillion et al and Maizlin et al. Management of each type of Amyand's hernia is well established according to the intraoperative circumstances. Type 1 hernias are treated using elective hernia repair with or without appendectomy.

The use of a prosthetic mesh can yield improved longevity of the repair. Surgical treatment for type 2 hernias is appendectomy and hernia repair, but mesh repairs are not recommended because of the increased risk of infection. A surgical approach for type 3 cases often relates to the extent of complications, which frequently require laparotomy. Thorough preoperative evaluation including CT of type 4 hernias can potentially detect the coexisting condition, avoid delay, help to plan the most appropriate procedure and thus improve the outcome. It is reported that perforated Amyand's hernia.[12] with a scrotal abscess is an extremely rare condition and is often misdiagnosed as a strangulated inguinal hernia/testicular torsion/testicular vascular embolism, which also represents a surgical emergency. Use of preoperative CT allows planning of proper surgical treatment to avoid complications and prevent sepsis.

In the presence of symptoms of GI dysfunction, the radiological and surgical evidence (On inguino-scrotal exploration) of purulent collection in the hernial sac provides a clue to the presence of intra-abdominal source of sepsis. Appendicectomy with drainage of purulent abdominal and scrotal collections under appropriate antibiotic coverage results in satisfactory recovery.

\section{CONCLUSION}

Acute appendicular perforation in young patients into the scrotum in a preformed hernia sac of indirect variety is though uncommon, it is not rare. The appearance of scrotum was suggestive of torsion of testes and patient feels unbearable pain in the scrotum. Proper investigation can easily diagnose the case and appropriate treatment in the earliest part can cure the disease of acute hemiscrotum and sequel. Mesh should not be applied in hernia repair when appendicular perforation in the sac detected. Formal appendicectomy with appropriate toileting of the peritoneal cavity is the treatment of choice when suspicious of contamination or features of peritonism.

\section{REFERENCES}

1. Charissis G, Vlahakis I, Vlahakis S, et al. Right acute hemiscrotum caused by insertion of an inflamed appendix. Br Jr Urol 2002;89(9):967-8.

2. Roberto Méndez-Gallart, Manuel Gómez Tellado, Candal J, et al. Acute scrotum: an exceptional presentation of an acute non-perforated appendicitis in childhood. J Paediatrsurg 1998;33(9):1435-6. 
3. Singh S, Adivarekar P, Karmarkar SJ. Acute scrotum: in children: a rare presentation of acute, non-perforated appendicitis. Paediatr Surg Int 2003;19:298-9.

4. Friedman SC, Sheynkin YR. Acute scrotal symptoms due to perforated appendix in children: case report and review of literature. Paediatr Emerg Care 1995;11(3):181-2.

5. Shyam B Sharma, Vipul Gupta. Acute appendicitis presenting as acute hemiscrotum in a boy. Indian Jr of Gastroenterology 2004;23(4):455-7.

6. Salemis NS, Nisotakis K, Nazos K, et al. Perforated appendix and periappendicular abscess within an inguinal hernia. Surgery 2009;109:677-99.

7. Constantine S. Computed tomography appearances of Amyand hernia. J Comput Assist Tomogr 2009;33(3):359-62.
8. Alia CD, LoSchiavo MG, Tonante A, et al. Amyand's hernia; case report and review of the literature. Hernia 2003;7(2):89-91.

9. Sharma H, Gupta A, Shekhawat NS, et al. Amyand's hernia: a report of 18 consecutive patients over a 15-year period. Hernia 2007;11(1):31-5.

10. Pellegrino JM, Feldman SD. Acute appendicitis in an inguinal hernia: a case report. Gastroenterol 2009;5(1):49-55.

11. Johari HG, Paydar S, Davani SZ, et al. Left-sided Amyand hernia. Ann Saudi Med 2009;29(4):321-2.

12. House MG, Goldin SB, Chen H. Perforated Amyand's hernia. South Med J 2001;94(5):496-8. 